\title{
Bifocal metanephric adenoma
}

\begin{abstract}
Metanephric adenoma (MA) is a rare benign renal tumor. Diagnostic imagings do not elucidate this diagnosis versus renal carcinoma. We report the case of a woman with history of non-Hodgkin lymphoma. CT scan showed nodules in one kidney, and radical nephrectomy was performed under the appearance of hypernephroma. Two lesions were identified, and the histopathological diagnosis was MA. No other MA has been reported in patients with non-Hodgkin lymphoma. The correct diagnosis of this tumor prevents radical nephrectomy. In the current report we describe two foci of tumor, and being MA usually solitary, its benign characteristics might be more challenging.
\end{abstract}

Keywords: metanephricadenoma kidney, bifocal, bifocal renalneoplasm
Volume 4 Issue 2 - 2017

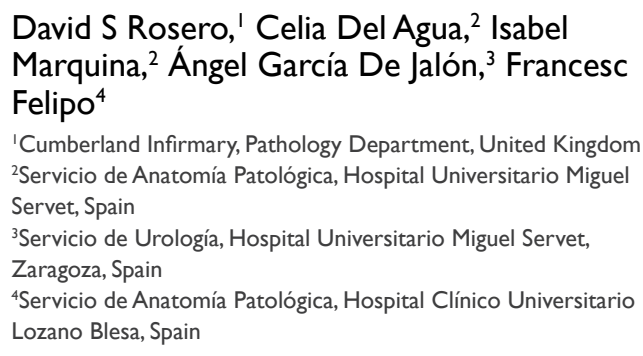

Correspondence: David S. Rosero, Pathology Department, Cumberland Infirmary, Newtown Rd, Carlisle, CA2 7HY United Kingdom, Tel +44I 2288I 4044, Email David.Rosero@ncuh.nhs.co.uk

Received: October 20, 2016 | Published: March 24, 2017

\section{Introduction}

Metanephric adenoma (MA) is a rare benign tumor of renal origin. ${ }^{1,2}$ First described by Mostofi in 1988, it represents $1 \%$ of renal neoplasms. ${ }^{3,4}$ The mean age of patients with MA is 41 years old, most commonly in the $5^{\text {th }}$ decade. ${ }^{5}$ Most of the MA-like tumors are detected incidentally by diagnostic imaging, without showing clinical findings. CT scans, ultrasound and MRI imaging do not usually further elucidate a specific image diagnosis of MA versus renal cell carcinoma. ${ }^{1}$ This concept is relevant, given that initially the malignancy of MA was uncertain, although it almost always has a benign clinical course. ${ }^{6}$ However, two cases have been reported showing malignant behavior with metastasis. ${ }^{6,7}$ MA ranges in size, being around $30-60 \mathrm{~mm}$ in diameter with the largest being $200 \mathrm{~mm} .{ }^{6}$ This tumor is usually unifocal and unilateral, with few exceptions reported, ${ }^{5,6}$

\section{Case presentation}

A 72-year-old woman with medical history of non-Hodgkin lymphoma in complete remission after chemotherapy underwent routine follow-up. Ultrasound and CT scan identified two echogenic masses in the right kidney. One solid, measuring $3.5 \mathrm{~cm}$; and the other cystic-like $3 \mathrm{~cm}$ in diameter. The solid lesion displayed an expansive pattern and hypernephroma appearance. A right radical nephrectomy was performed, under the prior clinical diagnosis of malignant neoplasm. The specimen revealed a $3.5 \mathrm{~cm}$ whitish circumscribed mass in the cortex in the lower pole. The cut surface made evident that the mass was separated by fibrous septae.
During the specimen sampling another solid nodular well- defined lesion was observed, located in the upper pole; a $0.8 \mathrm{~cm}$ diameter tumor with similar macroscopic features to those described in the former nodule. Furthermore, two renal retention cysts were found (measuring 3 and $1 \mathrm{~cm}$ diameter). Histologically, both solid tumors were composed of a highly cellular neoplasm (Figure 1) with basophilic proliferation of small rounded and extremely uniform cells with smooth nuclear contours, scant pale cytoplasm, dark-staining nuclei, and inconspicuous nucleoli. These tumoral cells were arranged into compact ductal and acinic fashion, with glomeruloid and papillary structures. Several psammoma bodies were also identified (Figure 1). Mitotic figures were absent throughout the lesion. The tumor expressed CK AE1AE3, CK18 and Vimentin (Figure 2). Besides, it was EMA, CK7 and WT-1 negative. The final diagnosis was MA.

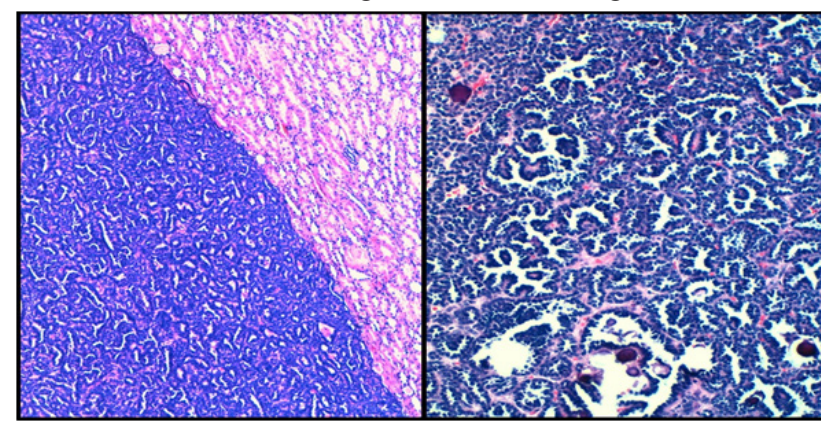

Figure I Well-defined and highly cellular neoplasm (left, HExI00). Several psammoma bodies and papillary structures were identified (right, HEx200). 


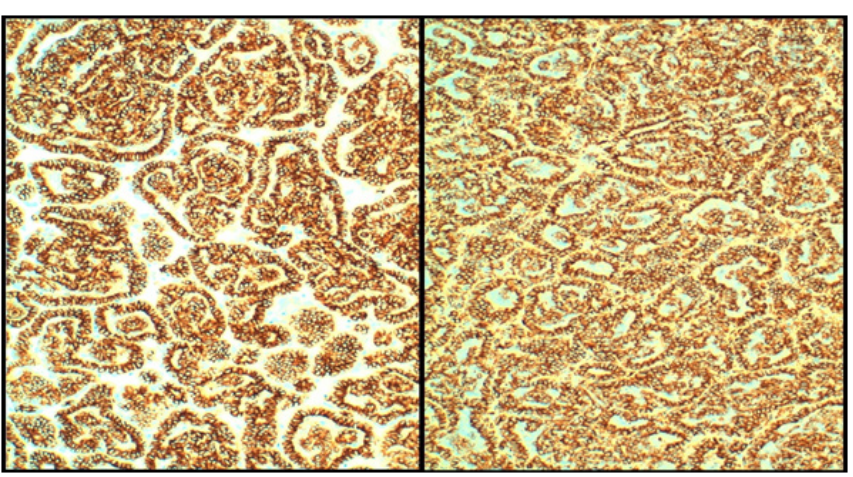

Figure 2 Tumoral expression of CKI 8 (left) and Vimentin (right). 200xs.

\section{Discussion}

MA is a rare kidney tumor. In the last 14 years only 2 cases of MA have been found among more than 100,000 biopsies reported at the Hospital Universitario Miguel Servet, Zaragoza, Spain. Some cases of MA have been described in the literature associated with hematological diseases, particularly polycythemia Vera. ${ }^{6,8}$ There is a higher incidence of simultaneous polycythemia compared to any other renal diseases. Besides, myelofibrosis and von Willebrand disease have been found in patients with MA. ${ }^{6}$ However, no other MA has been reported in patients with non-Hodgkin lymphoma diagnosed, as in our case. Until the present, it is still a casual relationship found.

The patient described above is older than the usual to MA; being 72 years old. However, patients with this tumor have been found in a wide range from 15 months to 83years old. ${ }^{5}$ Differential diagnosis includes papillary renal cell carcinoma, metanephric adenosarcoma, and metanephric stromal tumor, Wilms' tumor in adults and metastatic lung or papillary thyroid carcinoma. ${ }^{3}$ The accurate diagnosis of the usually benign tumor MA prevents unnecessary radical nephrectomy. Its clinical recognition may facilitate nephron-sparing surgery. ${ }^{1,3}$ In this matter we would like to remind that MA can be reliably diagnosed intraoperatively by frozen section, with histology mainly showing small, tightly packed cells. ${ }^{7}$ Precise preoperative characterization would help patient management. ${ }^{1,9}$ Metanephric adenomas are usually positive for WT-1., $, 7,9,10$ Nevertheless, in this case report WT-1 it was negative. Vimentin positive and the rest of the immunohistochemical profile was the usual for MA. Even though, there is no specific antibody profile for MA. ${ }^{6,9}$ For example, EMA and CK7 are absent in most MA, but some authors have reported positive results for these antibodies. ${ }^{5,9}$
MA is a rare benign tumor which has not been always well identified. The clinical and morphological features of this lesion should lead to accurate diagnosis, with the aim to deal better with the right treatment. In the current case report we found two tumoral foci, and being MA usually solitary, its benign characteristics might be more challenging, and may need some more clinical and radiological follow-up.

\section{Acknowledgements}

None.

\section{Conflict of interest}

The author declares no conflict of interest.

\section{References}

1. Masuda A, Kamai T, Mizuno T, et al. Renal metanephric adenoma mimicking papillary renal cell carcinoma on computed tomography: a case report. Urol Int. 2013;90(3):369-372.

2. Le Nué R, Marcellin L, Ripepi M, et al. Conservative treatment of metanephric adenoma. A case report and review of the literature. J Pediatr Urol. 2011;7(4):399-403.

3. Sharma PK, Tiwari P, Singh JP, et al. Metanephric adenoma: a case report and review of the literature. Saudi J Kidney Dis Transpl. 2013;24(5):1027-1030.

4. Snyder ME, Bach A, Kattan MW, et al. Incidence of benign lesions for clinically localized renal masses smaller than $7 \mathrm{~cm}$ in radiological diameter: influence of sex. J Urol. 2006;176(6):2391-2396.

5. Kohashi K, Oda Y, Nakamori M, et al. Multifocal metanephric adenoma in childhood. Pathol Int. 2009;59(1):49-52.

6. ain M, Rastogi A, Gupta RK. Atypical metanephric - a case report and review of literature. Int Urol Nephrol. 2007;39(1):123-127.

7. Terao H, Matsumoto T, Umemoto S, et al. Metanephric adenoma: report of two cases. Hinyokika Kiyo. 2008;54(9):599-602.

8. Yoshioka K, Miyakawa A, Ohno Y, et al. Production of erythropoietin and multiple cytokines by metanephric adenoma results in erythrocytosis. Pathol Int. 2007;57(8):529-536.

9. Patel NP, Geisinger KR, Zagoria RJ, et al. Fine needle aspiration biopsy of metanephric adenoma: a case report. Acta Cytol. 2009;53(3):327-331.

10. Kinney SN, Eble JN, Hes O, et al. Metanephric adenoma: the utility of immunohistochemical and cytogenetic analyses in differential diagnosis, including solid variant papillary renal cell carcinoma and epithelialpredominant nephroblastoma. Modern Pathology. 2015;28(9):1236-1248. 